Cómo citar este artículo en Chicago: Larison, Mariana. "Cuestiones fundamentales de fenomenología clásica: un acercamiento a su método y a sus posibles aplicaciones". Escritos 29, no. 62 (2021): 73-83. doi: http://dx.doi.org/10.18566/escr.v29n62.a05

Fecha de recepción: 26.01 .2021

Fecha de aceptación: 01.03.2021

\title{
Cuestiones fundamentales de fenomenología clásica: un acercamiento a su método y a sus posibles aplicaciones
}

\author{
Fundamental Issues of Classical Phenomenology: \\ An Approach to its Method and Possible Applications
}

\author{
Mariana Larison ${ }^{1}$ (1)
}

\begin{abstract}
RESUMEN
La fenomenología es una corriente filosófica que nace en los albores del siglo XX y continúa hasta nuestros días, enmarcada en un proyecto que toma forma durante el primer cuarto del siglo pasado, se diversifica en el segundo y prosigue múltiples caminos a partir de la década 1950. Resulta, por tanto, imposible hablar de "la" fenomenología, y necesario, en cambio, referirnos a un "proyecto" fenomenológico. En este artículo, ofrecemos un acercamiento a los problemas generales, la metodología y las posibles aplicaciones de la filosofía fenomenológica. En un primer momento, se abordará el sentido del proyecto fenomenológico general y el de tres de sus representantes clásicos y fundamentales: nos referimos a Husserl, Heidegger y Merleau-Ponty. En un segundo momento, buscaremos desplegar algunos de los conceptos más significativos en las propuesta de estos autores, destacando sus continuidades y rupturas. Finalmente, propondremos una ejemplo concreto de aplicación de la perspectiva y método fenomenológico a partir de nuestra experiencia contemporánea de la distancia corpórea.
\end{abstract}

Palabras clave: Fenomenología; Método; Edmund Husserl; Martin Heidegger; Maurice Merleau-Ponty; Distancia corpórea.

\begin{abstract}
Phenomenology is a philosophical perspective first established at the beginning of the twentieth century and remains being developed. It was shaped during the first quarter of that century, diversified during the second one, and took multiple roads since the 1950s. As a result, it is not possible to talk of 'the one' phenomenology, but instead it is necessary to speak of a phenomenological 'project'. The article presents an approach to the issues,
\end{abstract}

1 Doctora en Filosofía por la Université Paris 1 Panthéon-Sorbonne, Francia. Docente de la Facultad de Filosofía y Letras de la Universidad de Buenos Aires y en la Universidad Nacional de General Sarmiento, Argentina. Correo electrónico: malarison@gmail.com. 
method, and possible applications of phenomenological philosophy. First, it addresses the meaning of the general phenomenological project and that of the project of three of its classical representatives: Edmund Husserl, Martin Heidegger and Maurice Merleau-Ponty. Secondly, it introduces some of the key concepts of these philosophers, highlighting the continuities and breaks. Finally, it suggests a concrete example of a possible application of the phenomenological approach and method through our contemporary experience of corporeal distance.

Keywords: Phenomenology; Method; Edmund Husserl; Martin Heidegger; Maurice Merleau-Ponty; Corporeal Distance.

a fenomenología es una corriente filosófica que nace en los albores del siglo XX y continúa hasta nuestros días, enmarcada en un proyecto que toma forma durante el primer cuarto del siglo pasado, se diversifica en el segundo y prosigue múltiples caminos a partir de la década de 1950. Resulta, por tanto, imposible hablar de "la" fenomenología, y necesario, en cambio, referirnos a un "proyecto" fenomenológico. Un proyecto diseñado por el filósofo y matemático Edmund Husserl, quien, intrigado por la relación entre los aspectos subjetivos y objetivos de los procesos lógico-matemáticos de nuestro pensamiento, y sobre todo por su aparente contradicción, dedica las primeras décadas de su vida intelectual a elucidar y determinar el lazo que los vincula y los fundamenta.

Este lazo y los conceptos que lo despliegan sale a la luz de manera programática entre 1900 y 1901, con la aparición del primer y segundo tomo, respectivamente, de las Investigaciones lógicas. Esta obra funda la fenomenología como un modo de entender y abordar los fenómenos de consciencia, de la consciencia como lugar del aparecer de estos fenómenos, de las vivencias en las que tienen lugar y del carácter general de toda vivencia relacionada con un fenómeno objetivo, y así desarrolla la idea de una correlación subjetivo-objetiva que determina nuestra vida consciente: la intencionalidad. Sin embargo, esta obra no es todavía un proyecto filosófico determinado por un método, sino tal vez su ineludible manual conceptual-filosófico. Poco más de una década más tarde, en 1913, y luego de muchos trabajos publicados e inéditos, de cursos y de intercambio con colegas y estudiantes, aparece el libro que estructurará la fenomenología como filosofía primera y le dará un método, al que conoceremos en adelante como el método fenomenológico: se trata de Ideas relativas a una fenomenología pura y una filosofía fenomenológica. Libro primero: Introducción general a la fenomenología pura. A partir de esta obra, el trabajo husserliano no se detendrá y explorará hasta el fin de sus días los diversos aspectos que este método permite desplegar (la constitución de los fenómenos materiales, de los cuerpos vividos, de la persona, de la subjetividad trascendental, de la vida axiológica, de la vida intersubjetiva, entre otros), así como diversas modalidades de abordarlo (considerando, por ejemplo, los actos y sus objetos de manera estática o genética, es decir, su formación en el tiempo).

Paralelamente, otro filósofo alemán, discípulo dilecto de Husserl durante sus años de estudiante y colega poderoso más tarde, propone una nueva versión de la fenomenología como filosofía primera: Martin Heidegger expondrá, a fines de la década de 1920, una ontología fenomenológica que, a su manera, también busca elucidar la relación entre el mundo, las cosas y sus sentidos, por un lado, y por otro el existente, es decir, nosotros mismos en la medida en que comprendemos este sentido, lo interpretamos y lo interrogamos. Esta relación será caracterizada como trascendencia, y el método para su abordaje será la analítica existenciaria, que buscará describir y analizar las estructuras invariantes del existente 
y su mundo. En esta nueva versión, la puesta entre paréntesis de los prejuicios que exigía el método lo será ante todo de las determinaciones positivas del existente tanto como del sentido del ser. Esta nueva versión de la fenomenología tuvo un impacto enorme en la filosofía del siglo XX, e implicó algunas modificaciones y críticas muy profundas al método fenomenológico clásico.

En 1945, unas décadas después de la aparición de Ser y tiempo, y cuatro después de Ideas..., aparece en Francia, de la mano de Maurice Merleau-Ponty, Fenomenología de la percepción, una nueva perspectiva del proyecto y el método fenomenológico que radicaliza algunos aspectos poco explorados por la filosofía husserliana y propone un abordaje profundo del aspecto subjetivo de la correlación intencional o trascendencia: el cuerpo vivido. El problema de la relación entre el aspecto subjetivo y objetivo de los fenómenos, entre el existente que interroga e interpreta y el sentido de ser de lo que es, se plantea una vez más incorporando elementos de una y otra corriente, y desplegándose ahora bajo la forma de una relación entre el cuerpo propio vivido y lo sensible. Merleau-Ponty propone una lectura de la puesta entre paréntesis de los prejuicios sobre el aparecer y su reducción al campo fenomenal, característica del método fenomenológico, solo que el sentido de la reducción es menos la suspensión de la creencia en la independencia de la realidad del mundo respecto de nuestros actos constitutivos, o de las determinaciones positivas del ser y del existente, que la suspensión en la creencia de la verdad primera del discurso teórico sobre la experiencia pre-teórica. En la perspectiva merleau-pontiana, para comprender el cuerpo vivido tenemos que suspender los discursos de las diversas ciencias positivas como punto de partida y tomar, en cambio, nuestra experiencia perceptiva como inicio del análisis y la descripción fenomenal. Los discursos teóricos de la psicología (en sentido amplio) o la biología no alcanzan para dar cuenta de la experiencia concreta de nuestro cuerpo vivido.

En otras palabras, y como decíamos al comienzo, "la" fenomenología parece realizarse más bien como la permanente re-asunción de un proyecto y re-interpretación de un método, de los que nosotros, sus lectores, podemos por nuestra parte acercarnos a sus aspectos variantes y a aquellos a los que podríamos determinar, por qué no, invariantes.

\section{1. $I$}

La fenomenología como proyecto filosófico se instaura desde su origen en un diálogo con las ciencias de su época, principalmente las ciencias del hombre y fundamentalmente la psicología. Pero también se sitúa en una oposición principal respecto del campo científico: la oposición entre los hechos de los que se ocupan las ciencias empíricas y la esencia o sentido de estos hechos, de los que se ocupan las ciencias eidéticas, como la fenomenología. Mientras que unos, los hechos, se encuentran en campo de la experiencia empírica, reglada por leyes causales y las formas del espacio y el tiempo objetivos, los otros, los sentidos ideales, se sitúan fuera de estos, y responden a unas pocas leyes de estructura interna de alcance universal. La filosofía fenomenológica, que se presenta a sí misma como conocimiento del conocimiento, no puede desplegarse entonces en el campo de lo empírico, sino en el de lo eidético. Recordemos que el sentido del término eidético proviene del griego eidos: idea en el sentido de lo que es esencial, aquello sin lo cual algo no puede ser lo que es, pero también en el sentido de lo que está separado de lo sensible en un mundo de prototipos ideales. Pues bien, para la fenomenología husserliana, para comprender un hecho hace falta comprender su sentido, un sentido que guarde este doble aspecto del eidos y que la fenomenología propone analizar y describir en la medida en que no se está en el dominio de los hechos, sino en el dominio eidético del sentido. 
Desde el punto de vista husserliano, entonces, tanto los objetos de experiencia como la experiencia misma (cognoscitiva, pero también práxica, axiológica o afectiva), y las vivencias que son su estofa, no pueden comprenderse si no se comprende su estructura esencial, su sentido.

La fenomenología es, pues, un análisis descriptivo de los fenómenos, esto es, de lo que aparece a la consciencia tal como aparece, sin presuponer nada sobre el ser real de este aparecer, sino solo revelando el sentido que el fenómeno tiene para mí en el marco general de mi experiencia. Pero, vale aclarar esto una y otra vez, el ser para mí del sentido no implica un relativismo subjetivista, sino, por el contrario, la explicitación de estructuras y sentidos objetivos y comunes para todos los sujetos de experiencia consciente.

De esta forma, la fenomenología se erige, en el proyecto husserliano, en una ciencia descriptiva, de alcance universal, como el de toda ciencia estricta y con el rigor propio de toda ciencia rigurosa. La tarea de la fenomenología como filosofía descriptiva es la de aprehender, analizar y describir "el sentido que el mundo tiene para nosotros antes de todo filosofar", ${ }^{2}$ y podríamos agregar, antes de todo teorizar, tarea que solo puede realizarse, paradójicamente, a partir del filosofar o reflexionar.

Para esto, Husserl propone un método, el método descriptivo, que se realiza en diversos pasos. En primer lugar, se realiza una epojé, esto es, se pone entre paréntesis nuestra creencia en una realidad del mundo independiente de nosotros, del mundo y de todo lo que lo habita, para dejar ante la mirada reflexiva la pura correlación intencional, inscripta en el fenómeno que aparece, y describirlo tal como se da y dentro de los límites en los que se da.

En segundo lugar, los fenómenos deben ser descriptos en consideración a su doble carácter subjetivoobjetivo, a la vez actos o vivencias subjetivas, y sentido objetivo e ideal. Esto es precisamente lo que llamamos correlación intencional: una relación que encuentra en uno de sus polos actos subjetivos y en el otro sentidos objetivos. A esto llama Husserl descripción de la correlación noético-noemática, que puede concentrarse tanto en un polo como en otro de la correlación. Finalmente, la descripción así establecida es una descripción de esencias y de estructuras o relaciones esenciales. Es una descripción eidética en la medida en que describe la esencia del fenómeno. Estas esencias se obtienen a partir de una "reducción eidética", que se realiza a través de la variación imaginaria de un objeto hasta obtener de esta una invariante, aquello sin la cual el objeto dejaría de ser lo que es. La invariante nos da la esencia del objeto en una intuición (de esencias).

El sujeto que lleva adelante el método fenomenológico, el fenomenólogo o filósofo o persona reflexionante, es, en primera instancia, un yo empírico que, luego de practicar la epojé (suspensión) y poner en suspenso su creencia en el mundo, también pone en suspenso su yo empírico junto con este mundo. Solo nos quedamos con lo que aparece a la consciencia tal como aparece y con el correlato de este aparecer ya desligado de la creencia en el mundo: el yo trascendental o la subjetividad pura. Se nos revelará así en nosotros mismos un yo trascendental como el sujeto constituyente del sentido que encontramos en todo aparecer. La fenomenología se ocupa, entonces, del yo trascendental, del sujeto trascendental, que no se identifica con el hombre, sino con cualquier sujeto capaz de llevar adelante las operaciones de constitución de sentidos y que, a diferencia del hombre empírico y de las ciencias del hombre que son mudables según la época y el lugar, será un saber válido para cualquier sujeto racional.

2 Edmund Husserl, Meditaciones cartesianas (México: Fondo de Cultura Económica, 1996), 226. 


\section{II}

Heidegger, exponente de la segunda gran corriente dentro del proyecto fenomenológico, retoma las directrices de este, pero desde una perspectiva diferente de la husserliana. Para esta nueva versión, la fenomenología sigue identificándose con la filosofía en sentido general, pero un tal sentido general no será ya el conocimiento del conocimiento, sino el problema del ser de lo que es. El ser en sus múltiples sentidos e, incluso, el problema de la unidad de estos múltiples sentidos. El ser que no debe confundirse con el ente, con lo que de hecho es. En esta perspectiva, el método fenomenológico se configura en una peculiar versión ontológica.

Para Heidegger, Husserl comprendió que la tarea de la filosofía era ir a las cosas mismas para describirlas, pero la empresa quedó trabada por los supuestos idealistas y subjetivistas que la acompañaban: el supuesto de que el lugar privilegiado del análisis era la consciencia y de que la experiencia correspondía a un sujeto racional y universal, y no a un existente. Heidegger va a entender, en cambio, la fenomenología como una interrogación radical sobre lo que se muestra, tal como se muestra y no de los procesos conscientes que lo harían aparecer.

El primer paso es aquí también una "puesta entre paréntesis", pero de lo que se trata es de la suspensión de toda determinación positiva sobre el estatuto del existente, al que habitualmente nos referimos como "humano". El punto de partida de la interrogación heideggeriana es el ser humano, pero caracterizado meramente en tanto existente, es decir, no en el sentido de las ciencias positivas, no en el sentido de esta figura histórica a la que llamamos hombre, caracterizado con propiedades positivas (un cuerpo, una psiquis o un alma), sino el humano en tanto es un ente que existe y no que es, que está siempre fuera de sí, y en que tiene lugar la pregunta por el ser. A diferencia del resto de los entes, cuya esencia es determinada (ser mesa, ser silla, ser consciencia, etc.), la existencia del Dasein es abierta, nunca está concluida, su esencia es una tarea. ${ }^{3}$ La existencia aquí no es un modo de estar, sino un modo de "apertura hacia", cuyo contenido no viene dado previamente, sino que será realizada en cada caso por cada uno. Porque el Dasein es apertura, existencia, se encuentra siempre ligado al mundo en el que se encuentra: es ser-enel-mundo. Todo el análisis de Ser y tiempo será una elucidación de las estructuras de este ente particular y singular al que llama Da-Sein.

No se tratará en este caso de una variación de esencias, pero sí de la búsqueda de aquellas estructuras invariantes que constituyen al existente como tal, y a las que describirá y designará con el nombre de existenciarios. El carácter de salida fuera de sí del existente se denomina aquí trascendencia, y la correlación intencional se transformará así en relación de trascendencia entre un Dasein, su mundo y los entes que lo habitan. El sentido de este Dasein, de este mundo y de sus entes se complejiza, así como los procedimientos hermenéuticos para su comprensión en la medida en que el sentido deja de pensarse de manera ideal y se arraiga en estructuras existenciarias. El mundo humano específico en el que se encuentra el Dasein es un mundo práctico, afectivo, volitivo, compuesto de entes que poseen una significación para cada Dasein, y que mantienen entre ellos un reenvío de sentidos permanente: la silla reenvía a la mesa, esta a la comida, esta a... La totalidad del plexo de referencias y significaciones se revela finalmente, en esta descripción, como el sentido mismo del mundo.

3 Da-sein es un término alemán compuesto de da, "ahî", y sein, "ser", que adquiere un sentido técnico en la filosofía heideggeriana: señala lo esencial del existente, en tanto es el ente en que el ser se manifiesta, el ahí del ser, pues él es comprensión de ser. 


\section{III}

Pues bien, en la línea de estos desarrollos, pero marcando una nueva manera de entender la fenomenología, se destaca la figura del francés Maurice Merleau-Ponty. Del mismo modo que Heidegger, Merleau-Ponty se niega a reducir la experiencia humana a una relación entre la consciencia y sus objetos, y señala que, antes de todo comercio intelectual con el mundo, el hombre es una existencia abierta al sentido de las cosas que la rodean y de su mundo, un sentido que no es ideal y separable de los hechos, sino siempre un sentido encarnado, un sentido sensible. Pero, a diferencia de Heidegger, señala que es imposible hablar de estructuras invariantes de la existencia sin hacer referencia a la dimensión eminentemente corporal de todo existente humano y, podríamos decir, de todo viviente. El sujeto de la experiencia no es una consciencia, es cierto, pero tampoco una estructura vacía: es una corporalidad, al mismo tiempo sensible y sintiente, que no puede, bajo el pretexto de abandonar el orden de lo empírico, abandonar la corporalidad del sujeto.

El ser en el mundo es, ante todo, un ser corporal, y su modo originario de relación con las cosas, los otros y el mundo es una relación perceptiva. Realizar una analítica de la existencia a partir de una descripción fenomenológica de la experiencia vivida quiere decir para él describir e intentar comprender el sentido de la experiencia del cuerpo vivido tal como es vivido. La percepción no es una relación causal con el entorno, sino una interpretación del sentido del mundo por el cuerpo.

El viviente humano no es, como bien lo había señalado la fenomenología desde sus orígenes, un dato positivo, sino un fenómeno a ser interrogado, un fenómeno que se forma en el cruce de órdenes en apariencia divergentes o que se han pensado siempre de maneras divergentes: un cuerpo vivo y una consciencia, un alma y un cuerpo biológico, espíritu y materia. Pero el viviente humano, en tanto tal, permanece un misterio que orienta la investigación más que clausurarla. Es el nombre de un problema y no su respuesta.

La fenomenología, dirá Merleau-Ponty, no puede nunca abandonar completamente la actitud natural, como quería Husserl, si por esta se entiende aquella que mantiene un comercio con el mundo de la vida. Ningún conocimiento, ni siquiera el filosófico, puede darse fuera de esta relación originaria con el mundo, siempre situada desde una perspectiva y envuelta por determinaciones que no conoce o gobierna. Ni siquiera el conocimiento filosófico puede tener una visión desde las alturas, como en un sobrevuelo, como si no se encontrara enraizado en un suelo. Lo que sí puede hacer la filosofía es poner entre paréntesis la creencia, propia del conocimiento objetivo-teórico consistente en suponer que la relación verdadera con los objetos y con el mundo es una relación teórica desinteresada. En este sentido, es necesario poner entre paréntesis los prejuicios que interfieren con nuestra comprensión de la experiencia, de las cosas y de los otros dentro del mundo en que vivimos, tal como se nos dan y dentro de los límites en que se nos dan, principalmente los prejuicios que el discurso científico-objetivo instala como verdaderas objetivas y que obturan las relaciones del sujeto con su mundo. Pensar, por ejemplo, que nuestra experiencia del cuerpo vivido puede reducirse al discurso que la anatomía o la fisiología tienen sobre el cuerpo o a lo que la psicología dice sobre nuestro comportamiento. Pero someter a la crítica de la experiencia vivida las verdades científicas no quiere decir en ningún caso excluirlas de nuestras descripciones, sino simplemente excluir el prejuicio por el cual el mundo de la ciencia es el verdadero respecto del mundo de la experiencia vivida sobre el que, en definitiva, se erige y desarrolla. 
De esta forma, para dar cuenta de un fenómeno, ya sea natural, cultural, social o perceptivo, es necesario siempre dirigirse a la experiencia, a lo vivido, tal como ha sido vivido. Toda la filosofía merleau-pontiana se construye a partir de una profunda reflexión sobre el sentido filosófico de aquellos fenómenos que la ciencia o el conocimiento teórico describen, pero de los que no extraen sus consecuencias últimas: los casos de percepción patológica descriptos por la psiquiatría o la neurología nos enseñan el entrelazamiento de la vida afectiva y volitiva con la cognocitiva y con el pensamiento categorial; los desarrollos de la etología nos muestran cómo el comportamiento animal se rige por un universo simbólico habitualmente atribuido de forma exclusiva al animal-humano. Dicho de otro modo, nuestra experiencia, científica o cotidiana, nos enfrenta con una ontología que es necesario pensar, y, del mismo modo, con la ontología que describe el filósofo; si, en efecto, corresponde al mundo que habita, se expresa también en diversas modos de la experiencia.

Ahora bien, a partir de estas consideraciones generales sobre al menos tres maneras diversas de entender el proyecto y el método fenomenológicos, ¿qué podríamos tomar como núcleo común, como objetivos y problemas incesantes y presentes en sus representantes?

Podríamos considerar, por un lado, la necesidad de interrogar la experiencia en su doble vertiente, la del aparecer y la de aquel a quien aparece, la necesidad de problematizar cada uno de estos polos y de la relación misma. El método, en cada caso, es tanto descriptivo como analítico, apela siempre a la reflexión y realiza al mismo tiempo una crítica de esta reflexión, de sus diversos niveles y posibilidades. En todos los casos, se busca poner entre paréntesis aquella dimensión del aparecer que desligue este aparecer de su correlato subjetivo, que los vuelva indiferentes o independientes uno a otro, el acto y el sentido, la interpretación y el sentido, el cuerpo sintiente y el sensible.

Si tomamos entonces estos aspectos en cierta medida invariantes en los primeros proyectos formadores de la filosofía fenomenológica, ¿cómo podríamos pensar nosotros una aplicación de estos conceptos y principios?, ¿cómo podríamos, concretamente, "proceder entre fenomenólogos"?

Tomemos un fenómeno de nuestra experiencia reciente: la experiencia de la separación que nos tocó vivir en este último periodo de nuestras vidas signado por la pandemia. Todos hemos experimentado la separación, temporaria pero brutal, de nuestros afectos, de nuestros vínculos, de nuestros lugares, de nuestro mundo laboral o social. Tomemos, pues, esta experiencia e interroguémosla. Planteémosle una pregunta simple: hemos vivido la experiencia de la separación, ¿qué es lo que esta nos revela respecto de nosotros mismos, de las estructuras de nuestra experiencia y de nuestra subjetividad?

Elucidar esta pregunta supone avanzar sobre otras. Hemos vivido el esfuerzo por estar cerca, precisamente porque debíamos estar lejos. Pero ¿estuvimos lejos? En sentido estricto, estuvimos separados. Pero, entonces, ¿estar lejos y estar separados es lo mismo?, ¿es posible estar lejos estando juntos?, ¿o separados estando cerca?, ¿qué significa, en primer lugar, para nosotros, vivientes humanos, estar cerca? 
Porque, si cercanía y separación no son contradictorias entre sí, si estar lejos y estar juntos pueden constituir un mismo fenómeno, una misma situación, ¿por qué vivimos la separación como algo tan doloroso y extraño?, ¿por qué lo vivimos como una pérdida?

Para desplegar esta pregunta, necesitamos, entonces, comenzar por otra más simple: ¿qué significa estar cerca en relación con un cuerpo sensible y sintiente como el nuestro?

\section{2. $\mathbf{I}^{4}$}

Si procedemos como filósofo-fenomenólogos, es decir, intentamos analizar los fenómenos tal como se nos aparecen, para encontrar en ellos sus estructuras fundamentales, lo primero que tenemos que decir es que la cercanía y la lejanía son modos en que los objetos aparecen para nosotros. Pero este aspecto bajo el cual aparecen los objetos corresponde a ciertos tipos de objetos y no a todos: se trata de los objetos que se me ofrecen en persona y, en sentido estricto, lo que la fenomenología llama "en carne y hueso", es decir, de manera sensible, en su propia estofa: se trata de los objetos percibidos. La cercanía o la distancia de un objeto es un tipo de relación, bajo la que este aparece considerado respecto de un punto cero de referencia. En el caso de los objetos percibidos, el punto cero de referencia es el propio cuerpo del sujeto que percibe en una situación concreta, y el objeto dado en perspectiva, cercano o lejano respecto de mi cuerpo, es un objeto que aparece en persona, en carne y hueso, en tres dimensiones y dotado de algún tipo de materialidad sensible, indefectiblemente desde un lugar del espacio y del tiempo relativos a mi propia situación.

Este modo de darse del objeto percibido es diferente del modo en que se nos da, por ejemplo, el objeto en imagen: el árbol que percibo es diferente del árbol en imagen precisamente porque el árbol en imagen no puede variar la perspectiva en la que se me da, en la que lo veo, y esto no solo respecto de su cercanía o distancia, sino de sus lados o caras, no puedo recorrer su cuerpo con el mío y descubrir su parte trasera respecto de esta que veo ahora; no puedo acercarlo o alejarlo según el movimiento de mi cuerpo, el árbol de esta imagen será siempre ese objeto lejano del horizonte, no importa cuánto acerque la imagen a mi cuerpo.

La peculiaridad de la experiencia perceptiva nos liga para siempre a nuestro cuerpo sensible: solo percibe quien tiene un cuerpo como potencia motriz, y solo a partir de esta posibilidad de desplegar el espacio y experimentar lo cercano y lo lejano en relación con sí mismo es que la orientación en general tendrá sentido para ese sujeto. Del mismo modo que inaugura un espacio vivido, vemos que el cuerpo realiza también un movimiento temporal, una historia personal. Una historia hecha de recuerdos y expectativas, de experiencia más o menos cercanas, que configuran mi presente o brotan de él, también está hecha de un tiempo orgánico, de hábitos casi impersonales que co-participan de cada una de mis acciones conscientes. Una capa de habitualidades corpóreas, que no manejo y que no decido, pero que asumo y vivo, que constituyen mi estilo personal, forman como una dimensión casi impersonal en mi vida personal, del mismo modo que mi organismo completa mi estilo particular de caminar o ritmar mis palabras, de respirar y organizar mi existencia, y en general me plantea una y otra vez la pregunta fundamental sobre quién soy.

4 El análisis que sigue fue la base de la charla dictada en el ciclo virtual "La nuit des idées 2021", organizado por el Instituto Francés y la Embajada de Francia en Buenos Aires, del 28 de enero al 2 de febrero 2021. 
Cercanía y distancia, pues, son términos que cobran sentido para el viviente humano en la medida en que tiene un cuerpo sintiente y sensible, sin el cual ninguna de las relaciones de este tipo tendría en principio sentido.

\section{II}

Pero si ahondamos un poco más en este análisis, rápidamente vemos que, como el sentido de las palabras nos lo indicaba por su mero uso, proximidad y distancia no reenvían solo a la dimensión espaciotemporal de nuestra vida. Como decíamos al comienzo, la cercanía es un término que podemos aplicar tanto a las personas como a los lugares, a los seres queridos como a los objetos, tanto en sentido espacial como afectivo. La distancia espacial, o incluso temporal, no parecía ser contradictoria con la cercanía afectiva, que es el otro sentido del "estar cerca": puedo estar cerca de mi familia, de mis amigas y amigos, aunque estemos físicamente separados. Y puedo estar distante de la persona que camina a mi lado, que no conozco y no guarda ninguna cercanía, ninguna intimidad, conmigo.

Estar lejos no significa necesariamente, entonces, estar separados, ni estar cerca significa unívocamente estar juntos. Más aún, para algunos autores, es el carácter originariamente afectivo de la proximidad y la distancia lo que da un sentido derivado a la orientación espacial y temporal. Renaud Barbaras ha trabajado esta dimensión de una manera muy profunda en este pasaje que me permito citar:

La ciudad de Río de Janeiro se encuentra más próxima que la de Clermont-Ferrand, pues allí viven personas cercanas, hacia las que estoy dirigido y a las que visito cada vez que puedo. Sin embargo, la ciudad de Clermont-Ferrand, donde no voy casi nunca, sigue siendo próxima, es decir, se encuentra más cerca que tal ciudad situada a solo algunos kilómetros de aquí, pues allí fui profesor, porque allí se produjo un acontecimiento decisivo de mi existencia, que sigue allí, presente, es decir, en la proximidad, que el pasado al que me reenvía no está cerrado, mientras que esta ciudad cercana me resulta realmente extraña. Porque tampoco tengo nada que ver con ella y, por tanto, nada que hacer en ella. Pero, si ahora me sitúo a nivel del mundo y de las relaciones que lo rigen, diría que la distancia entre Buenos Aires y Río de Janeiro es poca y que son dos ciudades cercanas porque después de todo solo se encuentran a tres horas de avión y no a once, porque cuando estoy en Buenos Aires puedo visitar mis seres cercanos por la tarde, pero también, porque, a pesar de las grandes diferencias, son dos grandes ciudades de América Latina en las que encuentro un espíritu y una atmósfera comunes y donde me siento bien; en fin, dos ciudades cercanas. Vemos con este último ejemplo que son cercanas porque pertenecen, por decirlo de alguna manera, a un mismo espacio o, más bien, a una misma región o a un mismo lugar, pero solo pertenecen al mismo lugar porque son cercanas para mí, es decir, las dos forman parte de una región investida [por mí]: es este investimento lo que dibuja o circunscribe la región en la que puede haber proximidad. ${ }^{5}$

El investimento del que habla Barbaras establece una profundidad, una perspectiva respecto de mí que, lejos de reducirse a la dimensión espacial y temporal concretas, muestra el sentido derivado que las relaciones concretamente situadas guardarían respecto de una relación "primaria" con las cosas, las personas y, en general, el mundo: la relación afectivo-metafísica. En una perspectiva como la de Barbaras, las relaciones "meramente" espaciales y temporales no pueden comprenderse en toda su dimensión si no comprendemos primero el carácter profundamente metafísico de las nociones de proximidad y distancia,

5 Barbaras, Renaud. Introduction à une phénoméologie de la vie (París: Vrin, 2008), 319-320. 
y finalmente de "situación". El ser en situación, como bien lo describió Heidegger, no se reduce a la esfera de lo óntico, de lo que es, sino que debe buscarse en la de lo ontológico, en el del sentido de ser de lo que es. Más aún, todo su análisis de la existencia definida como-ser-en-el-mundo (análisis de una potencia hermenéutica innegable), se lleva a cabo sin hacer referencia a la dimensión sensible del sujeto de esta existencia, es decir, el viviente humano con su cuerpo concreto y sensible.

Podríamos decir, por tanto, siguiendo estos pensamientos, que estar lejos no significa necesariamente estar separados, ni estar cerca significa unívocamente estar juntos. En este mismo sentido, parece confirmarse nuestra época, en que el paradigma de la circulación es virtual más que corporal, en que las relaciones espaciales y temporales parecen haberse modificado completamente y sus respectivas relaciones también (entre ellas la de lo cercano y lejano), en que la posibilidad de la comunicación con lugares remotos es inmediata y el intercambio con espacios diversos tiene lugar en un mismo tiempo o el acceso a un mismo espacio virtual es posible en tiempos diversos.

\section{III}

Ahora bien, a pesar de que esto parece innegable, y precisamente porque en cierto modo lo es, resulta importante el llamado de atención que la misma experiencia impone, y repetir nuestra pregunta inicial. Si cercanía y separación no son contradictorias entre sí, si estar lejos y estar juntos pueden constituir un mismo fenómeno, una misma situación, ¿por qué experimentamos la separación de los cuerpos como lejanía?, ¿qué nos revela la separación respecto de la estructura de nuestra experiencia?

Del mismo modo que Merleau-Ponty, pero también que Husserl, diríamos que, para desentrañar este problema, es necesario volver una y otra vez a este punto cero de referencia de lo cercano y lejano en una situación vivida, al sujeto de la experiencia: nuestro cuerpo propio, indefectiblemente sensible y sintiente. Pues allí reside una clave importante de nuestro problema: volver al cuerpo sensible como punto cero de la experiencia no implica tanto establecer una prioridad de aspectos del cuerpo propio (espacio-temporal o afectivo, sensible o sintiente, perceptivo o imaginante), sino de entender la profunda imbricación e imposible separación entre estos aspectos si nos situamos en nuestra experiencia concreta. El cuerpo sintiente y sensible no se revela, como la filosofía y, en general, la cultura ha tendido a verlo, un elemento secundario respecto de mi vida personal, sino como su dimensión profunda. La percepción, ese modo en que un cuerpo vivido se abre al mundo, no se comprende si separamos nuestra sensibilidad abstractamente en una dimensión sensitiva, afectiva o estética. La sensibilidad como capacidad perceptiva se revela como una unidad, y como nuestra relación fundamental al mundo, sin la cual la experiencia de los objetos, de las personas y del mundo se reduce a una mera abstracción teórica de nuestra experiencia. Y esa sensibilidad, tomada en su dimensión unitaria, está siempre referida a un otro sensible.

Separados porque no podemos estar corporal y sensiblemente cerca, porque no podemos abrazarnos, besarnos, tocarnos, escupirnos al hablar de tan cerca que nos encontramos, compartir con las manos las mismas superficies, la experiencia dela separación nos revela esa cercanía fundamental, aquella que vivimos sin verla, algo como la estructura misma de nuestra vida: la experiencia de nuestra intercorporeidad. El estar referido a del cuerpo sensible se inscribe en la estructura de un mundo sensible, pone en evidencia nuestra existencia sintiente, nuestra corporeidad como intercorporeidad. Podemos decir en este sentido que la dimensión profundamente afectiva, incluso metafísico-afectiva, de la proximidad y la distancia a la que se dirigen posiciones como la heideggeriana o barbarasiana, no debe confundirnos respecto de 
la intuición fenomenológica primera: si bien la afectividad no se reduce a los cuerpos en su situación meramente sensible, la afectividad no tiene sentido sin ellos, no se entiende sin ellos.

El virus parece haber resaltado hasta qué punto nuestro mundo contemporáneo, más que cualquier otro, está hecho de un contacto de los cuerpos y sus fluidos, frente a la tendencia creciente a creerlo hecho de espacios y circulaciones virtuales, en dos dimensiones y desligadas de la presencia corpórea. En este sentido, se comprende la gravedad de nuestra pandemia, pues no es tanto su carácter virulento, sino la rapidez de su circulación el problema que plantea, y esa rapidez es correlativa del grado de contacto de los cuerpos unos con otros, rapidez que pone de manifiesto nuestro modo de existir como cuerpos tendidos hacia otros cuerpos. Y la separación que vivimos sea tal vez tan desgarradora porque nos revela eso que una y otra vez tendemos a olvidar: que la fragmentación del viviente humano de su cuerpo, de su cuerpo orgánico personal e interpersonal, cuerpo siempre sensible, es desgarradora.

Buscando desentrañar qué es aquello que debemos poner entre paréntesis, cuál es aquel prejuicio que, como algo que va de suyo, determina el sentido de nuestra experiencia y deja ver, en cambio, lo que esta misma experiencia nos revela, siguiendo su sentido y la subjetividad que los vivencia, la fenomenología, proyecto comenzado hace más de un siglo, todavía tiene mucho que enseñarnos para pensar la experiencia de nuestra contemporaneidad.

\section{Bibliografía}

Barbaras, Renaud. Introduction à une phénoménologie de la vie. París: Vrin, 2008.

Heidegger, Martin. Ser y tiempo. Buenos Aires: Fondo de Cultura Económica, 2009.

Husserl, Edmund. Investigaciones lógicas. Madrid: Alianza, 1982.

Husserl, Edmund. Ideas relativas a una fenomenología pura y una filosofía fenomenológica. México: Fondo de Cultura Económica, 1949.

Husserl, Edmund. Meditaciones cartesianas. México: Fondo de Cultura Económica, 1996.

Merleau-Ponty, Maurice. Fenomenología de la percepción. México: Fondo de Cultura Económica, 1957.

Larison, Mariana. L'être en forme: Phénoménologie, dialectique et ontologie dans la dernière philosophie de MerleauPonty. Milán: Mimesis, 2016.

Vigo, Alejandro. “ ¿Temporalidad y trascendencia? La concepción heideggeriana de la trascendencia intencional en 'Sein und Zeit"'. Acta Philosophica 6, n.o 1 (1997): 137-153.

Walton, Roberto J. Intencionalidad y horizonticidad. Bogotá: Aula de Humanidades, 2015. 